\title{
éditorial
}

\section{De la sexualité du bernard l'hermite}

Le règlement (CE) 1924(2006) du Parlement Européen et du Conseil concernant les allégations nutritionnelles et de santé portant sur les denrées alimentaires, publié au f.O. de l'Union Européenne du 18 janvier 2007, prend "comme critère d'évaluation le consommateur moyen normalement informé et raisonnablement attentif et avisé".

Il a été informé "normalement" par qui ce consommateur attentif? Par la presse? Pas par l'école assurément! Où sont les programmes de formation des maitres sur la santé en général et la nutrition en particulier? Il y a fort longtemps, à l'Association Française de Nutrition, avec A. François, nous avions essayé de sensibiliser l'Éducation Nationale à la possibilité de confier aux professeurs de sciences naturelles, on dirait aujourd'hui les professeurs de Sciences de la Terre, une approche des valeurs nutritionnelles des aliments. L'Inspecteur général délégué à cette réunion avait argué de la surcharge des programmes pour différer ce projet. À l'époque, mon fils, agé de 11-12 ans avait eu droit, en cours de sciences naturelles, à plusieurs heures consacrées à la reproduction du bernard l'hermite. Compte tenu des faibles chances que fean-Marc avait de rencontrer un bernard l'hermite, j'avais suggéré à cet excellent inspecteur de revoir les programmes pour les adapter au vécu des "consommateurs moyens". Ce qu'il n'avait pas apprécié. Ne croyez pas que cela ait beaucoup changé : discutant très récemment avec un responsable de la formation en IUFM, nous avons appris que l'éducation en matière de santé "ne faisait pas partie des priorités de l’Éducation Nationale.” 
Si l'Éducation Nationale ne nous permet pas d'apprendre, entre autres, bien entendu, à gérer notre capital santé, à quoi nous sert-elle? L'informatique, c'est bien, c'est l'avenir, c'est sur, mais seulement pour les gens vivants, et de préférence en bonne santé.

Quant à espérer que les industriels de l'alimentaire vont pouvoir combler, à coup de mentions obligatoires "indiquant l'importance d'une alimentation variée et équilibrée et d'un mode de vie sain (?) "l'absence de connaissances sur l'alimentation, il faut être singulièrement naîf. Les industriels de l'agro-alimentaire alléguent pour vendre. Et pour vendre leurs produits, pas ceux du voisin. Alors, leur demander de ne pas mentir, c'est normal, mais leur demander de faire de l'éducation générale, c'est déplacé.

L'éducation en nutrition, c'est aux Ministères de l'Éducation Nationale et de la Santé à la faire.

Comme disait Thomas fefferson, quand tout le monde sait lire, la presse est libre. C'est plus facile d'avoir l'esprit critique face aux publicités quand on sait de quoi il est question. On abuse plus facilement les gens mal informés :l'article 5 alinea 2 le dit bien "l'emploi d'allégations nutritionnelles et de santé n'est autorisé que si l'on peut s'attendre à ce que le consommateur moyen comprenne les effets bénéfiques exposés dans l'allégation". Comme on ne peut pas, raisonnablement, empêcher un fabricant d'exposer les qualités de ses produits, il serait urgent de faire en sorte que les consommateurs puissent comprendre ce dont on parle.

Monique ASTIER-DUMAS 